\title{
1 Ih blockade reduces cocaine firing patterns of putative 2 dopaminergic neurons of the ventral tegmental area in the 3 anesthetized rat
}

\author{
4 Karl Y. Bosque-Cordero ${ }^{1}$, Rafael Vazquez-Torres ${ }^{2}$, Cristhian Calo-Guadalupe ${ }^{2}$, Daisy Consuegra- \\ 5 Garcia $^{2}$, Giulia R. Fois ${ }^{3,4}$, François Georges ${ }^{3,4}$ and Carlos A. Jimenez-Rivera ${ }^{2}$ \\ 1 UPR Rio Piedras Campus, San Juan, PR. \\ 2 UPR Medical Sciences Campus, San Juan, PR. \\ 3 University of Bordeaux, Neurodegeneratives Diseases Institute, IMN-UMR-CNRS 5293, 146 \\ rue Léo Saignat, 33076 Bordeaux France. \\ 4 CNRS, Neurodegeneratives Diseases Institute, IMN-UMR-CNRS 5293, 146 rue Léo \\ Saignat, 33076 Bordeaux France
}

Abstract: The hyperpolarization-activated cation current $\left(\mathrm{I}_{h}\right)$ is a determinant of intrinsic excitability in various cells, including dopaminergic neurons (DA) of the ventral tegmental area (VTA). In contrast to other cellular conductances, $\mathrm{I}_{\mathrm{h}}$ is activated by hyperpolarization negative to $-55 \mathrm{mV}$ and activating $\mathrm{In}$ produces a time-dependent depolarizing current. Our laboratory demonstrated that cocaine sensitization, a chronic cocaine behavioral model, significantly reduces $\ln$ amplitude in VTA DA neurons. Despite this reduction in $\mathrm{I}_{\mathrm{h}}$, the spontaneous firing of VTA DA cells after cocaine sensitization remained similar to control groups. Although the role of $l_{h}$ in controlling VTA DA excitability is still poorly understood, our hypothesis is that $\mathrm{ln}$ reduction could play a role of a homeostatic controller compensating for cocaineinduced change in excitability. Using in vivo single-unit extracellular electrophysiology in isoflurane anesthetized rats, we explored the contribution of $I_{h}$ on spontaneous firing patterns of VTA DA neurons. A key feature of spontaneous excitability is bursting activity; bursting is defined as trains of two or more spikes occurring within a short interval and followed by a prolonged period of inactivity. Burst activity increases the reliability of information transfer. To elucidate the contribution of $\mathrm{I}_{\mathrm{h}}$ to spontaneous firing patterns of VTA DA neurons, we locally infused an $\ln$ blocker (ZD 7288, 8.3 $\mu \mathrm{M})$ and evaluated its effect. In blockade significantly reduced firing rate, bursting frequency, and percent of spikes within a burst. In addition, In blockade significantly reduced acute cocaine-induced spontaneous firing rate, bursting frequency, and percent of spikes within a burst. Using wholecell patch-clamp, we determine the progressive reduction of $\mathrm{l}_{\mathrm{h}}$ after acute and chronic cocaine administration (15mg/k.g intraperitoneally). Our data show a significant reduction ( $25 \%)$ in $\ln$ amplitude after 24 but not 2 hours of acute cocaine administration. These results suggest that a progressive reduction of In could serve as a homeostatic regulator of cocaine-induced spontaneous firing patterns related to VTA DA excitability.

Keywords: cocaine; ventral tegmental area; dopamine; electrophysiology; substance abuse disorder; rats; Ih current; neuronal excitability 


\section{Introduction}

Hyperpolarization-activated cation current $\left(\mathrm{I}_{\mathrm{h}}\right)$ is a significant modulator of intrinsic excitability on neurons of the mesocorticolimbic (MCL) system, including dopaminergic cells (DA) from the ventral tegmental area (VTA) [13]. Neuroadaptations in this network are hypothesized to trigger substance abuse disorder. In contrast to other ionic conductances, $I_{h}$ is a slowly activating cation inward current activated by hyperpolarizing inputs negative to $-55 \mathrm{mV}$. In activation depolarizes the membrane to a threshold level for the generation of action potentials [4]. Additionally, $\ln$ is involved in various neuronal properties such as the control and modulation of the rhythmic activity of neural circuits $[5,6]$, regulation of the resting membrane potential [7], firing frequency modulation [8,9], regulation of synaptic transmission $[10,11]$ and dendritic integration of synaptic inputs [12-14]. On VTA DA neurons, $I_{h}$ is classified as an excitatory driving force [15-18].

Neuromodulation of intrinsic properties is a decisive determinant of neuron excitability $[19,20]$. Intrinsic excitability is a measure of the neuron's inherent electrical properties that can be adjusted by numerous factors $[21,22]$. These adjustments arise through changes in morphological features and the expression level or biophysical properties of ion channels in the membrane [23]. The activity of a single neuron results from the summation of excitatory and inhibitory synaptic inputs, and the intrinsic membrane properties [24]. Neuroadaptations can be categorized as either homeostatic, that contribute to stabilizing neuronal excitability, or non-homeostatic, that produce an alteration from basal excitability $[19,25,26]$. Homeostatic changes in neuronal excitability can arise in response to long periods of altered activity [27-29]. For example, 3 hours after acute cocaine administration, activity of VTA DA neurons recorded in vivo, is significantly increased [30]. Our laboratory demonstrated that cocaine sensitization, a chronic cocaine behavioral model, significantly reduces $\ln$ amplitude by $\sim 40 \%$ in VTA DA neurons [31]. This reduction of current occurs concomitantly with the reduction of membrane surface $\mathrm{HCN} 2$ protein, the main $\mathrm{HCN}$ subunit in VTA DA neurons [32]. An important observation is that after the development of cocaine sensitization, the spontaneous firing of VTA DA cells remains similar to that of control groups [31]. These findings suggest that $I_{h}$ reduction could reduce cocaine-induced excitability as a homeostatic adaptation to regulate neuronal excitability of VTA DA neurons.

$\ln$ increases burst firing by enhancing rebound spiking after the disinhibition of VTA DA neurons [33]. Additionally, pharmacological blockade of this current on VTA DA neurons can significantly decrease (by $40 \%$ ) the firing rate in the majority of these neurons; this reduction is suggested to be behaviorally significant [34]. While this study indicates that the modulation of $\mathrm{Ih}$ can have a role in controlling VTA DA neuronal excitability, the relationship is still poorly understood. Hence, the study of $I_{h}$ modulation on spontaneous firing activity could lead us to elucidate its contribution to VTA DA neuronal excitability.

To determine if reduction of $\ln$ can alter basal VTA DA neuronal excitability, we evaluated the effect of $\ln$ blockade on spontaneous firing patterns using in vivo single-unit extracellular recordings. VTA DA neurons 
can be divided by their firing patterns, classified by the average firing rate and the percentage of spike within a burst (\%SWB) [35]. To investigate how $\mathrm{Ih}_{\mathrm{h}}$ modulates cocaine-dependent excitability, we measured $\mathrm{ln}$ using whole-cell patch-clamp electrophysiology, 2 and 24 hours after acute cocaine injection. Additionally, we determined the effect of $\ln$ blockade on cocaine-induced spontaneous firing patterns using in vivo single-unit extracellular recordings. We postulate that a progressive $I_{h}$ reduction serves as a homeostatic regulator to oppose cocaine-induced excitability in VTA DA neurons.

\section{Results}

2.1. Effect of blockade of $I_{h}$ on in vivo anesthetized single-unit spontaneous VTA DA neuron firing.

To evaluate the effect of $I_{h}$ blockade on in vivo anesthetized spontaneous firing, we used double barrel pipettes [36]. These pipettes were comprised of a recording electrode and a perfusion pipette that contained ZD $7288(8.3 \mu \mathrm{M})$. We recorded a minimum of 100 seconds of baseline extracellular firing activity and then locally perfused at the vicinity of the recorded cell, 60nL of ZD 7288 to see the effect of $I_{h}$ blockade on neuronal firing patterns as seen on sample traces (Figure 1A). Local infusion of ZD 7288 significantly reduced the firing rate on VTA DA neurons (Figure 1B). The bursting frequency was also significantly reduced by $\ln$ blockade (Figure $1 \mathrm{C}$ ). This finding provides the first evidence that $I_{h}$ modulates the bursting activity of VTA DA neurons. Moreover, the percent of spikes in bursts was also significantly decreased by $\ln$ blockade (Figure 1D). The mean inter-spike intervals (ISI) from each recorded neuron were analyzed. As shown in (Figure 1E), the naïve group mean ISI was significantly increased after ZD 7288 local infusion (paired t test, $t=3.075$, df $=19 ; n=8$ rats $c=20$ cells). When comparing the total population of the naïve cells' with gaussian best fit for mean ISI, we found a significant increase from $0.1025 \pm 0.02122 \mathrm{~s}$ to $0.1750 \pm 0.02497 \mathrm{~s}$ after ZD 7288 infusion (Figure 1F). On (Figure 1G), a representative cell ISI histogram of the naïve group was plotted. Gaussian best fit values resulted in an increase baseline mean ISI from $0.0797 \pm 0.0003$ s to $0.2128 \pm 0.0046$ s after ZD 7288 infusion. These results suggest that $I_{h}$ blockade can significantly reduce firing patterns in VTA DA neurons in part due to an increase in mean ISI. Additionally, through $\mathrm{I}_{\mathrm{h}}$ modulation, we could control the firing activity of VTA DA neurons. 

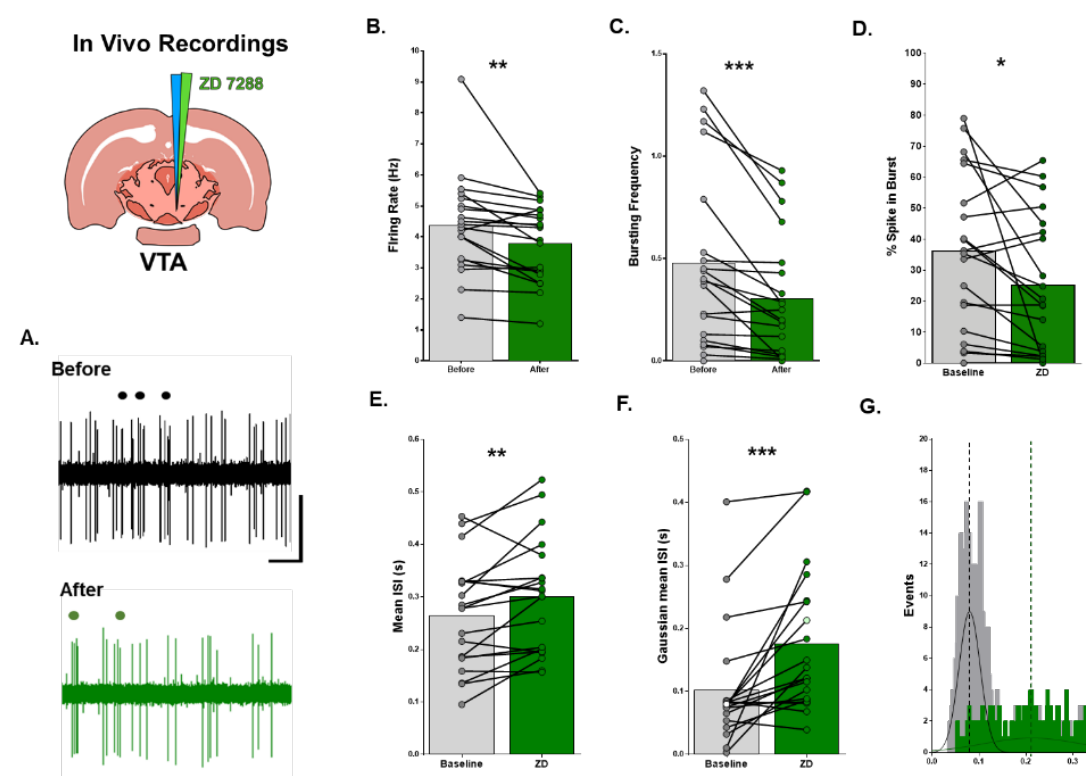

E.

F.
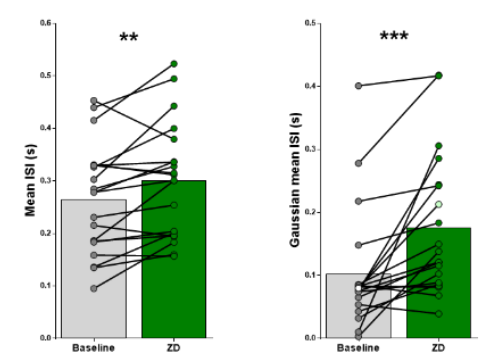

G.

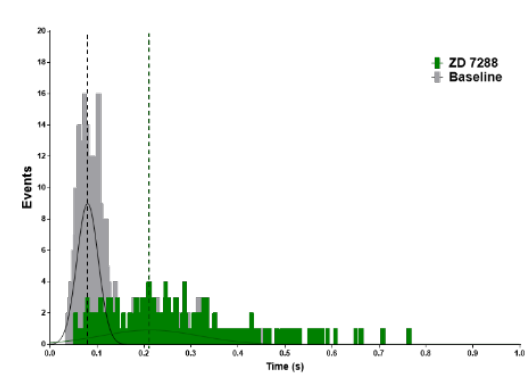

127

128

129

130

131

132

133

134

135

136

137

138

139

140

141

142

143

144

145

146

147

148

149

150

151

\section{7}

Figure 1. $I_{h}$ blockade significantly reduced firing properties on in vivo extracellular recordings of VTA DA neurons. 1A. Sample traces before and after $I_{h}$ blocker ZD 7288 (8.3um) perfusion. Each dot represents a burst event. Scale bars: horizontal, $1 \mathrm{~s}$; vertical, $1 \mathrm{mV}$. 1B. $\mathrm{I}_{\mathrm{h}}$ blockade significantly reduced the firing rate (paired $t$ test, $\mathrm{t}=2.912$ $\mathrm{df}=19$ ). 1C. $I_{\mathrm{h}}$ blockade significantly reduced bursting frequency (Wilcoxon signed-rank test). 1D. Ih blockade also significantly reduces the percent of spikes in bursts (paired $t$ test, $t=2.428 \mathrm{df}=19$, rats $\mathrm{n}=8$, cells $=20$ ). 1E. Mean Interspike interval was significantly increased after ZD 7288 infusion (paired $t$ test, $\mathrm{t}=3.075 \mathrm{df}=19$, rats $\mathrm{n}=8$, cells=20). 1F. Gaussian fitting mean ISI significantly increased after ZD 7288 infusion. 1G. A representative naive cell ISI histogram. Gaussian best fit values resulted in an increase baseline mean ISI from $0.0797 \pm 0.0003$ s to $0.2128 \pm$ 0.0046 s after ZD 7288 infusion. *Asterisk denotes significant differences $(p<0.05)$.

\subsection{Effect of Ih blockade on VTA DA neuron firing modes.}

Dopaminergic neurons of VTA can be categorized by the average firing rate and the percentage of spike within a burst (SWB; number of spikes within burst). These firing pattern classifications are based on characteristic interspike interval distribution. Low firing neurons (LF) fire at rates $<5 \mathrm{~Hz}$, while High firing neurons $(\mathrm{HF})$ fire at frequencies $>5 \mathrm{~Hz}$. Low Bursting Activity is denoted as \%SWB lower than $20 \%$, while High Bursting Activity is classified as \%SWB higher than $20 \%$ for neurons with firing rates $<5 \mathrm{~Hz}$ and $\%$ SWB higher than $40 \%$ for neurons with firing rates of $>5 \mathrm{~Hz}$ [35]. On (Figure 2A,2B), the naïve populational firing modes before and after ZD 7288 perfusion are plotted. Chi-squared analysis demonstrated significant difference in the HFHB and HFLB groups after ZD 7288 perfusion ( $p<0.05$; $X 2$ test) (Figure 2C.). These results suggest that $\mathrm{l}$ blockade is sufficient to change basal firing mode categories. 
A.

Before ZD 7288

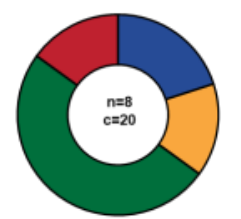

B. Before ZD 7288

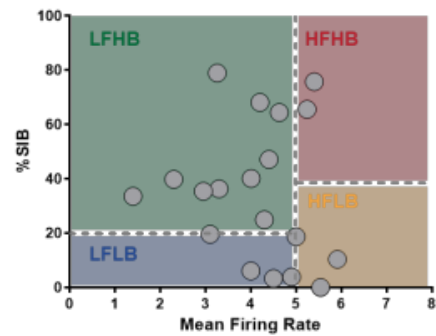

After ZD 7288

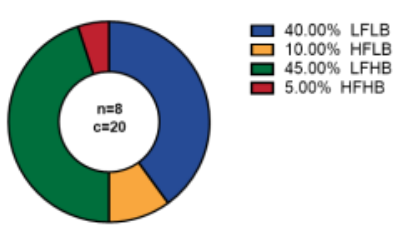

After ZD 7288

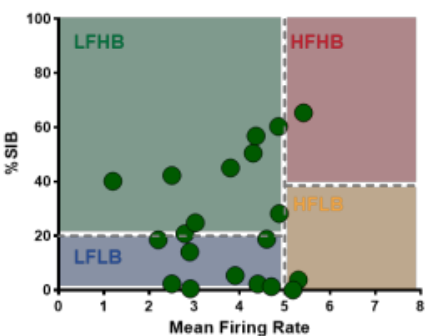

C. Naïve Group: Firing Modes

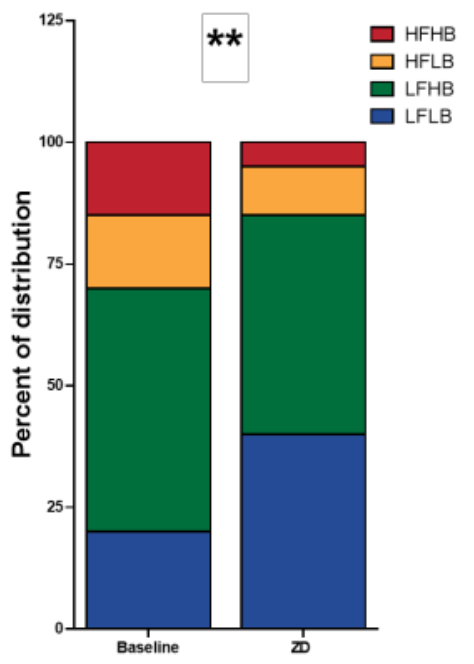

Figure 2. Effect of $I_{h}$ blockade on VTA DA neuron firing modes. 2A. Pie chart of naïve group firing mode population before and after ZD 7288. 2B. Mean Firing rate vs percent of spikes in burst of each recording. Colors represent lighter versions of the ones depicted in the pie charts. $2 \mathrm{C}$. Naïve group firing pattern percent of distribution demonstrates a significant difference in the HFHB and HFLB groups after ZD 7288 ( $p<0.05 ; x 2$ test).

\subsection{Effect of $I_{h}$ blockade on in vivo extracellular firing patterns 24 hours} after cocaine administration.

Using whole-cell patch-clamp voltage-clamp protocol we, evaluated $\mathrm{Ih}$ amplitude 2 and 24 hours after acute cocaine injection $(15 \mathrm{mg} / \mathrm{kg})$ and after chronic ( 7 days) cocaine administration (cocaine sensitization) (Figure 3A). 2 hours after acute cocaine, there is no significant difference in In amplitude compared to control. However, 24 hours after acute cocaine, we found a significant reduction in $\ln$ amplitude ( 25\%) in DA VTA neurons from cocaine sensitized rats. Note that after cocaine sensitization there was also a significant reduction in $\mathrm{lh}$ amplitude ( 40\% (Figure 3A) and [31]). Mechanistically, the continuous $I_{h}$ reduction after cocaine sensitization could serve as a homeostatic adaptation for neuronal excitability of VTA DA neurons.

Acute cocaine administration significantly increases firing activity of in vivo VTA DA neurons [30]. In blockade decreases firing rate through an increase in rebound delay and interspike interval $[17,33,34,37]$. Thus, In blockade may reduce cocaine-induced enhanced in vivo spontaneous activity. To evaluate the effect of $\mathrm{l}$ blockade on spontaneous firing of DA VTA neurons after acute cocaine administration in anesthetized rats, we used double barrel micropipettes. These pipettes are composed of a recording glass electrode (1$2 \mu \mathrm{m}, 10-12 \mathrm{M} \Omega$ filled with $2.0 \%$ pontamine sky blue in $0.5 \mathrm{M}$ sodium acetate

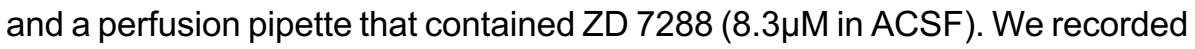
a minimum of 100 seconds of baseline extracellular firing activity and perfused 
$60 \mathrm{~nL}$ of $\mathrm{ZD} 7288$ to record the effect of $\ln$ blockade on the firing patterns induced by acute cocaine as seen on sample traces (Figure 3B). Post perfusion of ZD 7288 significantly reduced the cocaine-induced firing rate on VTA DA neurons (Figure $3 \mathrm{C}$ ). The cocaine-induced bursting frequency was also significantly reduced by $I_{h}$ blockade (Figure 3D). Additionally, cocaineinduced percent of spikes in bursts was also significantly decreased by $\mathrm{I}_{\mathrm{h}}$ blockade (Figure 3E). These findings suggest that $l_{h}$ blockade can significantly reduce cocaine-induced firing patterns of VTA DA neurons. The mean ISI from each recorded neuron were analyzed. As shown in (Figure 3F), the acute cocaine group mean ISI was significantly increased after ZD 7288 local infusion (paired $t$ test, $t=3.393$, df $=9 ; \mathrm{c}=10$ cells). When comparing the acute cocaine population with gaussian best fit for mean ISI, we found a significant increase from $0.1339 \pm 0.01499$ to $0.1942 \pm 0.03328 \mathrm{~s}$ after $\mathrm{lh}$ blockade (Figure 3G). On (Figure 3H), a representative cell ISI histogram of the cocaine group was plotted. Gaussian best fit values resulted in an increase baseline mean ISI from $0.0935 \pm 0.0013$ s to $0.2034 \pm 0.0069$ s after ZD 7288 infusion. These findings suggest that $I_{h}$ blockade can significantly reduce acute cocaine-induced firing patterns in VTA DA neurons in part due to an increase in mean ISI.
In Vitro Recording

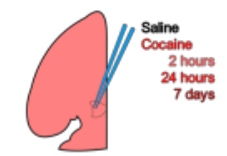

A.

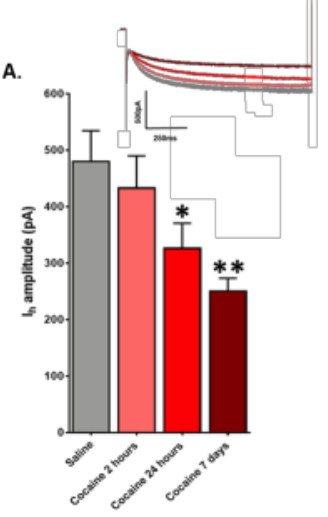

In Vivo Recording

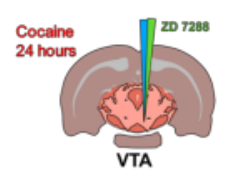

B.
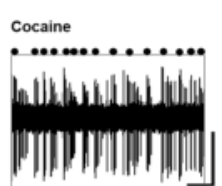

After
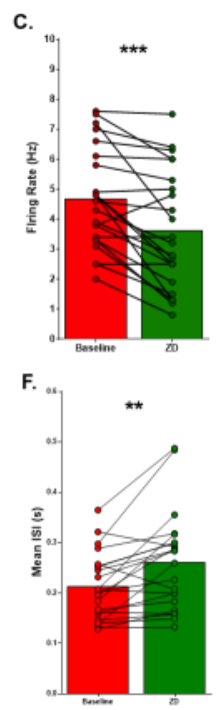
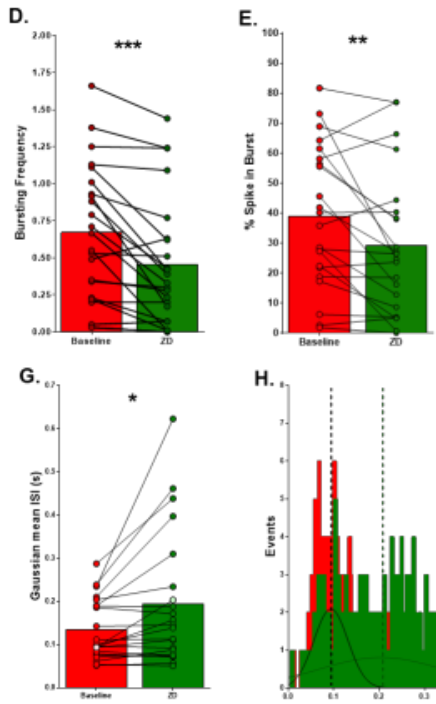

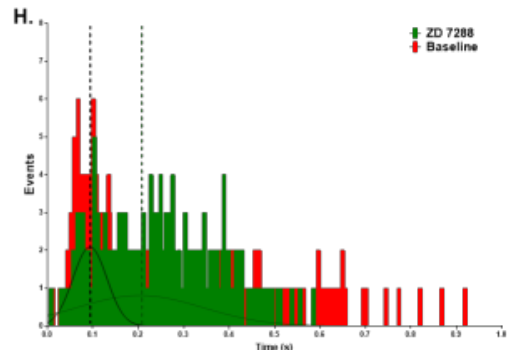

Figure 3. Post perfusion $I_{h}$ blockade significantly reduced firing properties after acute cocaine on in vivo extracellular recordings of VTA DA neurons. 3A. Whole cell patch-clamp voltage-clamp protocol $\mathrm{l}_{\mathrm{h}}$ amplitude 2 and 24 hours after acute cocaine injection $(15 \mathrm{mg} / \mathrm{kg})$ and after chronic (7 days) cocaine administration. A significant $\mathrm{Ih}_{\mathrm{h}}$ reduction occurs 24 hours after acute cocaine administration. One-Way ANOVA, Newman-Keuls multiple comparison test $F(3,42)=5.659$. 3B. Sample traces of acute cocaine injection and after $I_{h}$ blocker $Z D 7288$ (8.3um) perfusion. Each dot represents a burst event. Scale bars: horizontal, $1 \mathrm{~s}$; vertical, $1 \mathrm{mV}$. 3C. In blockade significantly reduced the cocaine-induced firing rate (paired $t$ test, $\mathrm{t}=4.633 \mathrm{df}=22$ ). 3D. $\mathrm{l}$ blockade significantly reduced cocaine-induced bursting frequency (paired $t$ test, $\mathrm{t}=4.383 \mathrm{df}=22$ ). $3 \mathrm{E}$. $\mathrm{ln}$ blockade also significantly reduces the percent of spikes in bursts. (paired $t$ test, $\mathrm{t}=2.428 \mathrm{df}=19$, rats $\mathrm{n}=5$, cells $=23$ ). 3F. Mean Interspike interval was significantly increased after ZD 7288 infusion. 3G. Gaussian fitting mean ISI significantly increased after ZD 7288 infusion. 3H. A representative cell ISI 
histogram. Gaussian best fit values resulted in an increase baseline mean ISI from $0.0935 \pm 0.0013$ s to $0.2034 \pm$ 0.0069 s after ZD 7288 infusion. *Asterisk denotes significant differences $(p<0.05)$.

\subsection{Effect of $I_{h}$ blockade on acute cocaine-induced VTA DA firing modes.}

Since VTA DA neurons can be categorized by the average firing rate and the percentage of spike within a burst, we tested if ZD 7288 infusion can alter acute cocaine treated animals firing modes. On (Figure 4A, B), the acute cocaine populational firing modes before and after ZD 7288 perfusion are plotted. Chi-squared analysis demonstrated no significant difference in any category after ZD 7288 infusion (Figure 4C.). Although Ih blockade can reduce acute cocaine-induced firing patterns, these results suggest that $\ln$ inhibition is not sufficient to alter the acute cocaine firing mode categories.
A. Before ZD 7288

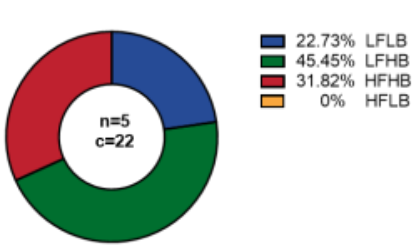

B. Before ZD 7288

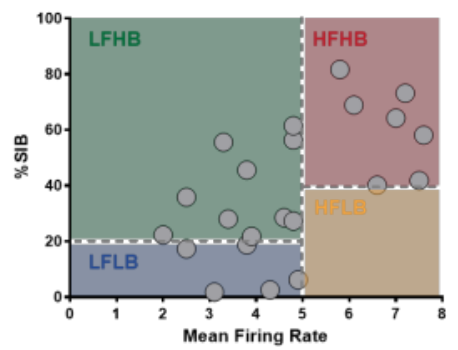

After ZD 7288

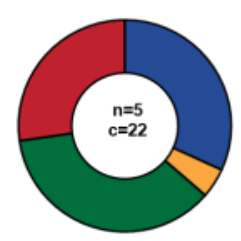

After ZD 7288

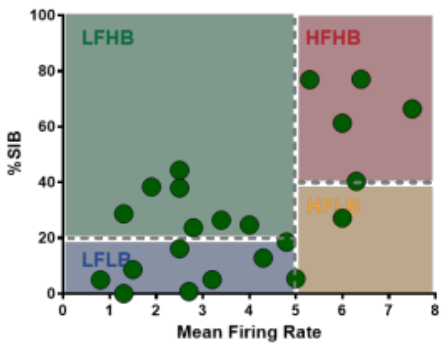

\section{Cocaine Group: Firing Modes}

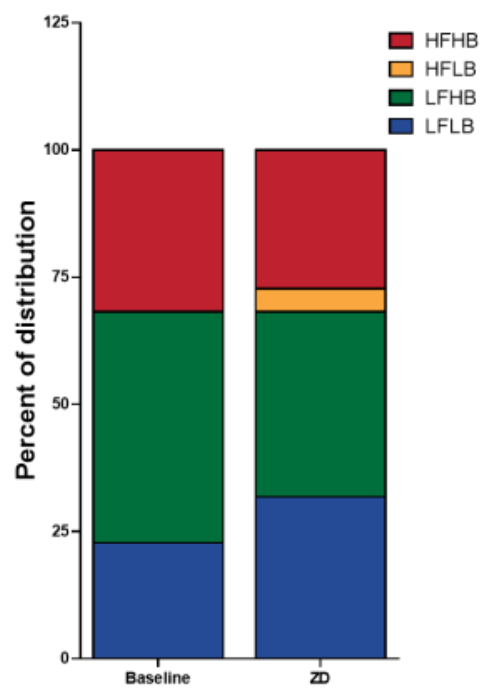

Figure 4. Effect of $I_{h}$ blockade on acute cocaine induced VTA DA firing modes. 4A. Pie chart of cocaine group firing mode population. 4B. Mean Firing rate vs percent of spikes in burst of each neuron recorded in the cocaine group. Colors represent lighter versions of the ones depicted in the pie charts. 4C. Cocaine group firing pattern percent of distribution did not demonstrate significant differences in groups after ZD $7288(p<0.05 ; X 2$ test).

\section{Discussion.}

We have previously shown that cocaine sensitization reduces $\ln$ in VTA DA neurons [31]. In the present study we report that $I_{h}$ blockade reduces spontaneous firing parameters in VTA DA neurons of rats. We elucidated the contribution of $\ln$ to spontaneous activity using in-vivo extracellular recording in anesthetized rats. Through local infusion of ZD 7288 from a micropipette in the vicinity of the recording electrode, we determined that $\ln$ blockade significantly reduces VTA DA neurons firing rate and bursting activity. Moreover, this diminution in activity is due to an increase in the inter-spike interval induced by $\ln$ blockade. In addition, we explored the contribution of $\ln$ 
to changes in firing patterns induced by acute cocaine. Infusion of an $\mathrm{ln}$ blocker locally into the VTA of rats treated with acute cocaine significantly decreased cocaine-induced firing activity. Both spontaneous firing activity and percent of spikes within bursts was significantly reduced after $\ln$ blockade. In addition, $\mathrm{Ih}$ blockade in this group also increased its inter-spike interval. Finally, we measured the $I_{h}$ amplitude in VTA DA cells after an acute cocaine injection. We observed that a significant $l_{h}$ reduction occurs 24 hours after drug administration. These results reinforce our earlier observation that $\mathrm{ln}$ is reduced after cocaine sensitization, and suggest that a progressive reduction of $I_{h}$ current after cocaine exposure is a possible homeostatic regulator of VTA DA neuronal excitability.

Neuromodulation of intrinsic properties is a significant determinant of neuronal excitability $[20,21]$. Intrinsic excitability can be modified by altering the neuron's inherent electrical properties $[22,23]$. These adjustments in excitability arise through changes in morphological features and the expression level or biophysical properties of ion channels in the membrane [24]. The final response of a single neuron emerges through the summation of the of effects of excitatory and inhibitory synaptic inputs, and intrinsic membrane properties [25]. Homeostatic changes in neuronal excitability can arise in response to long periods of altered activity [27-29]. Such periods of altered activity may be a consequence of drug exposure.

The $\ln$ current modulates electrophysiological properties of neurons in the Mesocorticolimbic (MCL) system, including DA neurons of the VTA, in a variety of ways. Neuroadaptations in this network are hypothesized to contribute to the neuropathology of substance abuse disorder [38,39]. Dopaminergic neurons of the VTA are important elements that are activated by rewarding experiences [40-42]. In addition, alteration of normal functionality in the VTA underlies aspects of drug abuse and other neuropsychiatric disorders [43-46]. One of the most explored reward-related circuits is the VTA DA projection to the nucleus accumbens [47-49]. The alteration of normal dopamine neurotransmission by persistent drug administration can trigger both molecular and cellular mechanisms in this circuit that can lead to substance abuse disorder $[40,49,50]$.

Different studies have identified $I_{h}$ as an excitatory driving force in VTA DA neurons $[18,19]$. Pharmacological In blockade suppresses DA neuronal firing in both VTA and substantia nigra zona compacta neurons in vivo [33,34]. After pharmacological blockade, an artificially mimicking $I_{h}$ via current injection via dynamic clamp restored the normal intrinsic DA cell membrane potential phenotype [33]. In modulation has been demonstrated to compensate for alterations in CA1 pyramidal neuronal excitability. Chronic pharmacological enhancement or excitability deprivation triggers a bidirectional plasticity of $\mathrm{l} h$ and leads to regulation of both input resistance and intrinsic excitability [51]. This evidence supports the possibility that $\mathrm{In}$ modulation can alter VTA DA excitability.

In current participates in burst formation in various neurons [52,53]. For example, rhythmic burst firing in thalamic relay neurons arises mainly from the interaction of two dominant currents, $\ln$ and $T$ type calcium channels (Icat) [7]. The $I_{h}$ activation depolarizes the membrane potential towards threshold activating $\mathrm{I}_{\mathrm{cat}}$ and generating low-threshold $\mathrm{Ca}^{2+}$ spike. The generation of 
$\mathrm{Ca}^{2+}$ spikes activate a burst of fast $\mathrm{Na}^{+}$and $\mathrm{K}^{+}$dependent action potentials. This provides a possible mechanism for how $l_{h}$ could influence burst firing in VTA DA neurons. Our results show that spontaneous firing activity of VTA DA neurons is significantly reduced by in vivo delivery of an $\ln$ blocker (Figure 1). Bursting activity and percent of spikes within the burst were also reduced. We can speculate that a similar mechanism of action as in the thalamus might be acting in the VTA for the generation of bursting activity. Further studies should address this hypothesis.

VTA DA neurons can be categorized in four main modes of firing pattern with respect to the average firing rate and the percentage of spikes within a burst [35]. Each group is also associated with a characteristic ISI histogram distribution. In blockade affected all VTA DA neurons firing patterns. In blockade increases the afterhyperpolarization potential in cell-attached recordings of VTA DA neurons, indicating a physiological mechanism for the increase in ISI produced by these blockers [18]. Here we provide evidence that after the $I_{h}$ blockade there is an increase in the ISI while also reducing the quantity of spiking activity (Figure 1 and 2). These results lead us to postulate that $\ln$ modulation in VTA DA neurons is important for the homeostatic regulation of excitability.

Drugs of abuse such as cocaine can induce acute and sustained changes in DA neuronal firing activity. A single exposure to cocaine increases firing rate and bursting activity of VTA DA neurons [30] concomitantly with a synaptic potentiation of these cells [54,55]. Furthermore, after the development of cocaine sensitization, a model of chronic cocaine exposure, the spontaneous firing rate of VTA DA cells remains similar to control groups. In these neurons, cocaine sensitization is associated with a reduction in $\mathrm{lh}$ amplitude by $\sim 40 \%$ [31]. The observed current reduction coincides with a HCN2 membrane surface protein diminution, the main HCN subunit in VTA DA neurons [32]. In addition, I current reduction persists after a 7-day cocaine withdrawal period [13]. Our evidence demonstrates a significant $(\sim 25 \%)$ reduction in $\ln$ amplitude 24 but not 2 hours after acute cocaine administration (Figure 3A). It has been demonstrated that an increase in bursting activity appears beginning at 24 hours following an acute cocaine injection [30]. High frequency stimulation of VTA DA neurons can also trigger a significant reduction in $I_{h}$ amplitude [56]. Thus, we hypothesize that cocaineinduced burst firing could trigger $\ln$ modulation as a homeostatic regulator to oppose the alteration of excitability induced by cocaine.

In Fig 2. we evaluated the Ih blockade effect on naïve animals VTA DA recordings. The chi-squared analysis demonstrated a significant difference in the HFHB and HFLB groups after ZD perfusion ( $p<0.05 ; x 2$ test). These results suggest that Ih blockade is sufficient to change the firing mode of VTA DA subpopulations within this category system. On the other hand, in Fig 4. we evaluated the effect of Ih blockade on acute cocaine-treated rats. This group did not have any significant change in subpopulation firing modes. We hypothesize this could be because VTA DA neurons in the acute treated group fire more frequently. Under these circumstances, an Ih blockade would not be sufficient to change the cells' firing mode category. Although, when comparing the population mean ISI on the acute treated group, we can detect a significant increase in the time between spikes. 
Our experiments demonstrate that 24 hours after a single cocaine injection, Ih blockade significantly reduces cocaine-induced firing and bursting activity. Similar to our first set of experiments, pharmacological blockade of $\mathrm{ln}$ reduces the acute cocaine-induced increase in spontaneous firing and bursting activity by increasing the mean ISI. This provides support for the idea that reduction of $\mathrm{l}_{\mathrm{h}}$ conductance could serve as a regulator of cocaine-induced excitability. Given the fact that the mean ISI on both naïve and acute cocaine groups significantly increased after ZD 7288, we propose that the reduction in firing patterns on both groups could be in part mechanistically due to the increase in time between spikes.

Our data demonstrated statistically significant differences on firing rate, bursting frequency and percent of spike in bursts. (Figure 1 bcd and Figure 3cde). Fundamental studies have established that DA neuronal baseline activity is irregular with an average firing rate of $4.5 \mathrm{~Hz}$ [57]. Our results demonstrate that after ZD 7288 local infusion there was a significant reduction in firing rate of approximately $1 \mathrm{~Hz}$ in both naïve and acute cocaine groups. We suggest that the reduction in firing rate of a normally low firing DA neuron could be enough to alter cellular functionality. Indeed, it has been demonstrated that optogentically-induced phasic firing on VTA DA neurons is sufficient to establish behavioral conditioning [58]. While optogeneticallyinduced tonic firing on VTA DA neurons decreases sucrose intake [59]. It has been demonstrated that burst formation provides a new platform of neuronal coding used to enhance the gain of neural signaling by increasing transmitter release and improving synaptic potentiation [60]. Bursts also enhance the reliability of information transfer and provide various stimulus features in the same spike train [61]. These studies provide evidence that our significant reduction in bursting activity and firing patterns after $\ln$ blockade could also lead to distinctive physiological and behavioral alterations.

Although previous evidence and our current results can define $\mathrm{Ih}_{\mathrm{h}}$ modulation as a possible homeostatic regulator of intrinsic excitability, it is one component of different interrelated biophysical properties that results in the regulation of spontaneous firing activity. For example, small conductance $\mathrm{K}+(\mathrm{SK})$ current amplitude is another determinant of endogenous DA firing patterns. Computational models evaluating VTA DA neurons firing patterns denote that high SK conductance is characteristic in low firing neurons, while low SK conductance is present in high firing neurons [62]. Further studies will evaluate the influence of other conductances in our experimental timepoints and how the integration and interplay of these diverse currents can alter VTA DA neuronal excitability.

Currently, little is known of how homeostatic regulation of neuronal excitability is initiated in the MCL system. We suggest these modifications could be triggered by cocaine-induced enhancement of glutamatergic excitatory afferent towards VTA DA neurons [54, 63]. Repeated exposure to cocaine can also disinhibit VTA GABA neurons thus increasing neuronal firing of VTA DA neurons [64]. Our hypothesis postulates that specific cocaineinduced enhancement of bursting patterns could result in alteration of $\mathrm{l}_{\mathrm{h}}$, since $24 \mathrm{hrs}$ after acute cocaine administration, bursting activity increases, and this is correlated with a significant reduction in $I_{h}$ current. Our evidence supports the idea that $I_{h}$ reduction could be a homeostatic regulator that reduces 
spontaneous firing rate after cocaine-induced firing patterns are present. In diminution can increase the ISI, thus reducing the probability of generating an action potential.

Previous studies have demonstrated that ZD 7288 can additionally block $\mathrm{Na}+$ currents in HEK293 cells transfected with Nav 1.4 plasmids [65]. This effect is unlikely to influence our results because local ZD 7288 infusions did not alter the action potential amplitude in our recordings. Studies have shown that isoflurane inhibits $\ln$ current in mice cortical pyramidal neurons [66]. Our experimental design evaluated both baseline and the effect of $\mathrm{l}_{\mathrm{h}}$ blockade on firing patterns from the same neuron of the anesthetized rat. We found that prior isoflurane alteration of $\mathrm{l}_{\mathrm{h}}$ amplitude does not influence our experimental results after ZD 7288 infusions since the effect was only present after $I_{h}$ blockade. Moreover, our VTA DA neuronal baseline firing patterns were similar to other experiments using different types of anesthetics such as chloral hydrate and sodium pentobarbital as well as in awake behaving animals [57, 67-69].

In sum, we conclude that $\ln$ blockade can decrease the spontaneous firing activity of VTA DA neurons. Furthermore, $\ln$ blockade can also reduce the acute cocaine-induced increase in spontaneous firing activity in VTA DA cells. It was found that the reduction in firing activity can be correlated with an increase in the inter-spike interval. In addition, there was a significant reduction in $\mathrm{l}_{\mathrm{h}}$ current $24 \mathrm{hrs}$ after cocaine administration. We have previously discovered that after the development of cocaine sensitization $\mathrm{ln}_{\mathrm{h}}$ is significantly reduced whereas the spontaneous firing activity of VTA DA cells remains unaltered [31]. Thus, the progressive current reduction suggested here from acute to chronic cocaine administration could serve as a homeostatic mechanism to reduce cocaine enhanced firing patterns. The study of the regulatory role of this current can provide novel insights on how changes in intrinsic neuronal properties might establish cellular homeostasis in the VTA DA system.

\section{Experimental Procedure:}

\subsection{Animals}

Procedures involving experimental animals were be performed according to the U.S. Public Health Service Publication Guide for the Care and Use of Laboratory Animals and were approved by the Animal Care and Use Committee at the University of Puerto Rico Medical Sciences Campus. Behavioral and electrophysiological experiments were performed with male Sprague-Dawley rats (35-51 days postnatal). Animals were housed two per cage and were maintained at constant temperature and humidity with a 12:12$\mathrm{h}$ light-dark cycle. Water and food were provided ad libitum.

\subsection{Whole-cell Patch-Clamp Electrophysiology.}

Midbrain horizontal slices $(220 \mu \mathrm{m})$ containing the VTA were prepared from male Sprague Dawley rats (35-51 days postnatal) as previously described [70]. Whole-cell voltage- and current-clamp recordings were obtained from visually identified neurons in the VTA with an infrared microscope with differential interference contrast (DIC) (BX51WI Olympus). 
Putative DA neurons were identified by the presence of the $I_{h}$ and were located lateral to the fasciculus retroflexus and medial to the medial terminal nucleus of the accessory optic tract (MT) [71]. Margolis et al. [72,73] clearly demonstrated that every cell that expresses tyrosine hydroxylase $(\mathrm{TH})$ also coexpresses $I_{h}$, and hence every VTA DA cell displays $I_{h}$. The converse argument, however, is not always true, i.e., if $\mathrm{I}_{\mathrm{h}}$ is present in a VTA cell then it may not be dopaminergic [63]. Nonetheless, we recorded from medial VTA neurons lateral to the MT, where other authors have reported that $\ln$ and $\mathrm{TH}$ are colocalized in $\sim 75 \%$ of cells [74]. Therefore, the contribution of non-DA cells to the data shown here is likely to be minimal. Recording pipettes (borosilicate glass, OD $1.5 \mathrm{~mm}$, ID $1.0 \mathrm{~mm}$; WPI, Sarasota, FL) had a resistance of 3-5 $\mathrm{M} \Omega$ when filled with (in $\mathrm{mM}$ ) $115 \mathrm{KCH} 3 \mathrm{SO} 4$ (methyl potassium sulfate), $20 \mathrm{KCl}, 1.5 \mathrm{MgCl} 2,5 \mathrm{HEPES}, 1 \mathrm{EGTA}, 2 \mathrm{ATP}, 0.2 \mathrm{GTP}$, and 10 creatine phosphate (CP), pH 7.25, 290 mOsm. (Na)GTP, (Mg)ATP, and $(\mathrm{Na}) \mathrm{CP}$ were added fresh daily. Artificial cerebrospinal fluid (ACSF) contained (in mM) $127 \mathrm{NaCl}, 2.5 \mathrm{KCl}, 1.25 \mathrm{NaH} 2 \mathrm{PO} 4,25 \mathrm{NaHCO}, 2 \mathrm{CaCl}$, $1 \mathrm{MgCl}$, and $25 \mathrm{D}(+)$-glucose, and were equilibrated with 95\% O2 5\% CO2 at $35^{\circ} \mathrm{C}$. Data sets were collected through an Axopatch 200B amplifier (Axon Instruments; Molecular Devices, Sunnydale, CA), digitized at $5 \mathrm{kHz}$, filtered at $1 \mathrm{kHz}$, and stored in a computer using pCLAMP 9 (Axon Instruments; Molecular Devices). Series resistance was monitored during the entire recording, voltage-clamp data were discarded if changes $>15 \%$ in series resistance occurred.

\subsection{In Vivo Anesthetized Single-Unit Neuron Recordings.}

Male Sprague Dawley Rats were anesthetized with $4 \%$ of isoflurane 2 $\mathrm{l} / \mathrm{min}$ air and $\mathrm{O}_{2}$ for induction and placed in the stereotaxic frame. During the surgical procedures and neuronal recordings, $1.5 \%$ isoflurane $2 \mathrm{l} / \mathrm{min}$ air and $\mathrm{O}_{2}$ were delivered through a facial mask via spontaneous respiration. Body temperature was maintained between $36-38^{\circ} \mathrm{C}$ with an animal temperature controlled electric heating pad during the procedure (World Precision Instruments, Sarasota, FL). A glass micropipette (1-2 $\mu \mathrm{m}, 10-12 \mathrm{M} \Omega$ filled with $2.0 \%$ pontamine sky blue in $0.5 \mathrm{M}$ sodium acetate) were lowered into the VTA. A double barrel pipette was used, similar to the ones used by Georges and Aston-Jones [36]. The perfusion pipette on the double barrel pipette was filled with ZD $7288(8.3 \mu \mathrm{M})$ an $\mathrm{l}$ blocker disolved in ACSF. Electrodes were lowered in the anterior and lateral part of the VTA according to stereotaxic coordinates derived from rat brain atlas and corrected empirically (anteroposterior: -5.0-6.0 $\mathrm{mm}$ from bregma; mediolateral: 0.7-1.0 $\mathrm{mm}$ from midline; dorso-ventral: -7.8-8.4 mm from bregma). To distinguish dopamine from nondopamine neurons, the following parameters were used: (1) an action potential width greater than $1.1 \mathrm{~ms}$ (measured from the start of action potential to the negative trough); (2) slow spontaneous firing rate $(<10 \mathrm{~Hz})$; (3) single and burst spontaneous firing patterns (characterized by spikeamplitude decrement). The extracellular potential was recorded with an Axoclamp-2B amplifier and filter $(300 \mathrm{~Hz} / 0.5 \mathrm{~Hz}$ : Georges and Aston-Jones, 2002). Single-neuron spikes were collected online (CED 1401; SPIKE 2; 
Cambridge Electronic Design). A baseline of 100 seconds was recorded and then 100 seconds after delivery of $60 \mathrm{~nL}$ of the ZD 7288 solution.

\subsection{Data analysis.}

All data were presented as mean \pm SEM. The statistical significance of two group comparisons were assessed performing paired-sample Student t-test when data followed normal distribution or Wilcoxon matched-pairs test when data did not follow normal distribution. For multiple comparisons, data values were subjected to a one-way ANOVA followed by Newman-Keuls multiple comparison as a post hoc analysis. $P$ values were reported throughout the text and the significance was set at $p<0.05$.

Author Contributions: Conceptualization, K.Y.B.-C., R.V.-T. and C.A.J.-R.; methodology, K.Y.B.-C., R.V.-T. and C.A.J.-R.; validation, K.Y.B.-C., R.V.-T., C.C.G., D.C.-G., G.R.F., F.G. and C.A.J.-R.; formal analysis, K.Y.B.-C. and F.G.; investigation, K.Y.B.-C., C.C.-G., D.C.-G., G.R.F. and F.G.; resources, F.G. and C.A.J.-R.; data curation, K.Y.B.-C. and F.G.; writing-original draft preparation, K.Y.B.-C.; writing-review and editing, K.Y.B.-C., R.V.-T., C.C.-G., D.C.-G. and C.A.J.-R.; visualization, K.Y.B.-C.; supervision F.G. and C.A.J.-R.; project administration, K.Y.B.-C., F.G. and C.A.J.-R.; funding acquisition, F.G. and C.A.J.-R. All authors have read and agreed to the published version of the manuscript.

Funding: This research was funded by the National Institute of General Medical Sciences (GM084854), the National Center for Research Resources (5R25GM061838-15, 2G12-RR003051), the National Institute on Minority Health and Health Disparities (8G12-MD007600), the NSF Partnerships in International Research and Education (PIRE) Program Neural Mechanisms of Reward \& Decision (OISE-1545803), the Research Initiative for Scientific Enhancement RISE Program (5R25GM061151-18), the Centre National de la Recherche Scientifique (CNRS), the Universite de Bordeaux and by LABEX BRAIN ANR-10-LABX-43.

Acknowledgments: The authors thank Dr. Mark S. Brodie for the excellent proofreading and critiques of the manuscript. This work was in partial fulfillment of Karl Y. Bosque-Cordero doctoral dissertation as a requirement to obtain a Ph.D. degree from the University of Puerto Rico, Rio Piedras Campus.

Conflicts of Interest: The authors declare no conflict of interest. The funders had no role in the design of the study; in the collection, analyses, or interpretation of data; in the writing of the manuscript, or in the decision to publish the results.

\section{References}

1. DiFrancesco, Dario. "A study of the ionic nature of the pacemaker current in calf Purkinje fibres." J. Physiol. 314, no. 1 (1981): 377-393.

2. Adams, Paul R., and Halliwell, James V. "A hyperpolarization-induced inward current in hippocampal pyramidal cells." In J. Physiol. London, vol. 324, no. Mar, pp. P62-P63. 40 West 20th Street, New York, NY 10011-4211: Cambridge Univ Press, 1982.

3. Kase, Daisuke, and Keiji Imoto. "The role of HCN channels on membrane excitability in the nervous system." J. Signal Transduction (2012).

4. Tsantoulas, Christoforos, Elizabeth R. Mooney, and Peter A. McNaughton. "HCN2 ion channels: basic science opens up possibilities for therapeutic intervention in neuropathic pain." Biochem. Journal 473, no. 18 (2016): 27172736. 
5. Leresche, Nathalie, Dora Jassik-Gerschenfeld, Matthias Haby, Ivan Soltesz, and Vincenzo Crunelli. "Pacemakerlike and other types of spontaneous membrane potential oscillations of thalamocortical cells." Neurosci. Let. 113, no. 1 (1990): 72-77.

6. McCormick, David A., and Hans-Christian Pape. "Properties of a hyperpolarization-activated cation current and its role in rhythmic oscillation in thalamic relay neurones." J. Physiol. 431, no. 1 (1990): 291-318.

7. Doan, Tan N., and Kunze, Diana L. "Contribution of the hyperpolarization-activated current to the resting membrane potential of rat nodose sensory neurons." J. Physiol. 514, no. 1 (1999): 125-138.

8. Friedman, Allyson K., Jessica J. Walsh, Barbara Juarez, Stacy M. Ku, Dipesh Chaudhury, Jing Wang, Xianting Li et al. "Enhancing depression mechanisms in midbrain dopamine neurons achieves homeostatic resilience." Science 344, no. 6181 (2014): 313-319.

9. Okamoto, Takashi, Mark T. Harnett, and Hitoshi Morikawa. "Hyperpolarization-activated cation current $\left(\mathrm{I}_{\mathrm{h}}\right)$ is an ethanol target in midbrain dopamine neurons of mice." J. Neurophysiol. 95, no. 2 (2006): 619-626.

10. Beaumont, Vahri, and Robert S. Zucker. "Enhancement of synaptic transmission by cyclic AMP modulation of presynaptic I h channels." Nat. Neurosci. 3, no. 2 (2000): 133-141.

11. Sparks, Daniel W., and Chapman, Andrew. "Contribution of $I_{h}$ to the relative facilitation of synaptic responses induced by carbachol in the entorhinal cortex during repetitive stimulation of the parasubiculum." Neuroscience 278 (2014): 81-92.

12. Arencibia-Albite, Francisco, Rafael Vázquez-Torres, and Carlos A. Jiménez-Rivera. "Cocaine sensitization increases subthreshold activity in dopamine neurons from the ventral tegmental area." J. Neurophysiol. 117, no. 2 (2017): 612-623.

13. Engel, Dominique, and Vincent Seutin. "High dendritic expression of $\mathrm{I}_{\mathrm{h}}$ in the proximity of the axon origin controls the integrative properties of nigral dopamine neurons." J. Physiol. 593, no. 22 (2015): 4905-4922.

14. Magee, Jeffrey C. "Dendritic integration of excitatory synaptic input." Nat. Rev. Neurosci. 1, no. 3 (2000): 181190.

15. Santoro, Bina, and Mala M. Shah. "Hyperpolarization-activated cyclic nucleotide-gated channels as drug targets for neurological disorders." Ann. Rev. Pharmacol. Toxicol. 60 (2020): 109-131.

16. Zhong, Peng, Casey R. Vickstrom, Xiaojie Liu, Ying Hu, Laikang Yu, Han-Gang Yu, and Qing-song Liu. "HCN2 channels in the ventral tegmental area regulate behavioral responses to chronic stress." Elife 7 (2017): e32420.

17. Wanat, M. J., F. W. Hopf, G. D. Stuber, P. E. M. Phillips, and A. Bonci. "Corticotropin-releasing factor increases mouse ventral tegmental area dopamine neuron firing through a protein kinase C-dependent enhancement of $\mathrm{I}_{\mathrm{h}} . "$ J. Physiol. 586, no. 8 (2008): 2157-2170.

18. Neuhoff, Henrike, Axel Neu, Birgit Liss, and Jochen Roeper. "lh channels contribute to the different functional properties of identified dopaminergic subpopulations in the midbrain." J. Neurosci. 22, no. 4 (2002): 1290-1302.

19. Desai, Niraj S., Lana C. Rutherford, and Gina G. Turrigiano. "Plasticity in the intrinsic excitability of cortical pyramidal neurons." Nat.Neurosci. 2, no. 6 (1999): 515-520.

20. Nelson, Alexandra B., Claudia M. Krispel, Chris Sekirnjak, and Sascha Du Lac. "Long-lasting increases in intrinsic excitability triggered by inhibition." Neuron 40, no. 3 (2003): 609-620.

21. Kemenes, Ildikó, Volko A. Straub, Eugeny S. Nikitin, Kevin Staras, Michael O'Shea, György Kemenes, and Paul R. Benjamin. "Role of delayed nonsynaptic neuronal plasticity in long-term associative memory." Current biology 16, no. 13 (2006): 1269-1279.

22. O'Leary, Timothy, Mark CW van Rossum, and David JA Wyllie. "Homeostasis of intrinsic excitability in hippocampal neurones: dynamics and mechanism of the response to chronic depolarization." J. Physiol. 588, no. 1 (2010): 157-170.

23. Williams, Alex H., Timothy O'Leary, and Eve Marder. "Homeostatic regulation of neuronal excitability." Scholarpedia 8, no. 1 (2013): 1656.

24. Beck, Heinz, and Yoel Yaari. "Plasticity of intrinsic neuronal properties in CNS disorders." Nat. Rev. Neurosci. 9, no. 5 (2008): 357-369.

25. Howard, Allyson L., Axel Neu, Robert J. Morgan, Julio C. Echegoyen, and Ivan Soltesz. "Opposing modifications in intrinsic currents and synaptic inputs in post-traumatic mossy cells: evidence for single-cell homeostasis in a hyperexcitable network." J. Neurophysiol. 97, no. 3 (2007): 2394-2409.

26. Wijesinghe, Rajiv, and Aaron J. Camp. "Intrinsic neuronal excitability: implications for health and disease." Biomol. Concepts 2, no. 4 (2011): 247-259.

27. Turrigiano, Gina G. "Homeostatic plasticity in neuronal networks: the more things change, the more they stay the same." Trends Neurosci. 22, no. 5 (1999): 221-227. 
28. Turrigiano, Gina G., and Sacha B. Nelson. "Hebb and homeostasis in neuronal plasticity." Current opinion in neurobiol. 10 , no. 3 (2000): 358-364.

29. Keck, Tara, Taro Toyoizumi, Lu Chen, Brent Doiron, Daniel E. Feldman, Kevin Fox, Wulfram Gerstner et al. "Integrating Hebbian and homeostatic plasticity: the current state of the field and future research directions." Philosophical Transactions of the Royal Society B: Biological Sciences 372, no. 1715 (2017): 20160158.

30. Creed, Meaghan, Jennifer Kaufling, Giulia R. Fois, Marion Jalabert, Tifei Yuan, Christian Lüscher, Francois Georges, and Camilla Bellone. "Cocaine exposure enhances the activity of ventral tegmental area dopamine neurons via calcium-impermeable NMDARs." J. Neurosci. 36, no. 42 (2016): 10759-10768.

31. Arencibia-Albite, Francisco, Rafael Vázquez, María C. Velásquez-Martinez, and Carlos A. Jiménez-Rivera. "Cocaine sensitization inhibits the hyperpolarization-activated cation current I $\mathrm{h}$ and reduces cell size in dopamine neurons of the ventral tegmental area." J. Neurophysiol. 107, no. 8 (2012): 2271-2282.

32. Santos-Vera, Bermary, Ana del C. Vaquer-Alicea, Cristina E. Maria-Rios, Alan Montiel-Ramos, Aynette RamosCardona, Rafael Vázquez-Torres, Priscila Sanabria, and Carlos A. Jiménez-Rivera. "Protein and surface expression of HCN2 and HCN4 subunits in mesocorticolimbic areas after cocaine sensitization." Neurochem. Int. 125 (2019): 91-98.

33. Tateno, Takashi, and Hugh PC Robinson. "The mechanism of ethanol action on midbrain dopaminergic neuron firing: a dynamic-clamp study of the role of I h and GABAergic synaptic integration." J. Neurophysiol. 106, no. 4 (2011): 1901-1922.

34. Seutin, Vincent, Laurent Massotte, Michel-François Renette, and Albert Dresse. "Evidence for a modulatory role of $\mathrm{l}_{\mathrm{h}}$ on the firing of a subgroup of midbrain dopamine neurons." Neuroreport 12, no. 2 (2001): 255-258.

35. Mameli-Engvall, Monica, Alexis Evrard, Stéphanie Pons, Uwe Maskos, Torgny H. Svensson, Jean-Pierre Changeux, and Philippe Faure. "Hierarchical control of dopamine neuron-firing patterns by nicotinic receptors." Neuron 50, no. 6 (2006): 911-921.

36. Georges, François, and Gary Aston-Jones. "Activation of ventral tegmental area cells by the bed nucleus of the stria terminalis: a novel excitatory amino acid input to midbrain dopamine neurons." J. Neurosci. 22, no. 12 (2002): 5173-5187.

37. Migliore, Michele, and Rosanna Migliore. "Know your current $\mathrm{l}_{\mathrm{h}}$ : interaction with a shunting current explains the puzzling effects of its pharmacological or pathological modulations." PloS one 7.5 (2012): e36867.

38. Kauer, Julie A. "Addictive drugs and stress trigger a common change at VTA synapses." Neuron 37, no. 4 (2003): 549-550.

39. Chen, Kang, Ildiko Aradi, Niklas Thon, Mariam Eghbal-Ahmadi, Tallie Z. Baram, and Ivan Soltesz. "Persistently modified h-channels after complex febrile seizures convert the seizure-induced enhancement of inhibition to hyperexcitability." Nat. Med. 7, no. 3 (2001): 331-337.

40. Kauer, Julie A. "Learning mechanisms in addiction: synaptic plasticity in the ventral tegmental area as a result of exposure to drugs of abuse." Annu. Rev. Physiol. 66 (2004): 447-475.

41. Geisler, Stefanie, and Roy A. Wise. "Functional implications of glutamatergic projections to the ventral tegmental area." Reviews in the neurosciences 19, no. 4-5 (2008): 227.

42. Geisler, Stefanie, and Daniel S. Zahm. "On the retention of neurotensin in the ventral tegmental area (VTA) despite destruction of the main neurotensinergic afferents of the VTA-Implications for the organization of forebrain projections to the VTA." Brain Res. 1087, no. 1 (2006): 87-104.

43. Phillips, P. E., Stuber, G. D., Heien, M. L., Wightman, R. M., \& Carelli, R. M.. "Subsecond dopamine release promotes cocaine seeking." Nature, 422(6932), (2003): 614-618.

44. Wise, Roy A., and P-P. Rompre. "Brain dopamine and reward." Ann. Rev. Psychol. 40.1 (1989): 191-225.

45. Volkow, Nora D. "What do we know about drug addiction?." American J. Psychiatry 162, no. 8 (2005): 1401-1402.

46. Winterer, Georg, and Daniel R. Weinberger. "Genes, dopamine and cortical signal-to-noise ratio in schizophrenia." Trends in neurosciences 27, no. 11 (2004): 683-690.

47. Rice, Margaret E., and Jyoti C. Patel. "Somatodendritic dopamine release: recent mechanistic insights." Philosophical Transactions of the Royal Society B: Biological Sciences 370, no. 1672 (2015): 20140185.

48. Swanson, L. W. "The projections of the ventral tegmental area and adjacent regions: a combined fluorescent retrograde tracer and immunofluorescence study in the rat." Brain Res. Bulletin 9, no. 1-6 (1982): 321-353.

49. Everitt, Barry J., and Marina E. Wolf. "Psychomotor stimulant addiction: a neural systems perspective." J. Neurosci. 22, no. 9 (2002): 3312-3320.

50. Kauer, Julie A., and Robert C. Malenka. "Synaptic plasticity and addiction." Nat. Rev. Neurosci. 8, no. 11 (2007): 844-858. 
51. Gasselin, Célia, Yanis Inglebert, and Dominique Debanne. "Homeostatic regulation of h-conductance controls intrinsic excitability and stabilizes the threshold for synaptic modification in CA1 neurons." J. Physiol. 593, no. 22 (2015): 4855-4869.

52. Hughes, Stuart W., David W. Cope, and Vincenzo Crunelli. "Dynamic clamp study of $\mathrm{I}_{\mathrm{h}}$ modulation of burst firing and $\delta$ oscillations in thalamocortical neurons in vitro." Neuroscience 87, no. 3 (1998): 541-550.

53. Ying, Shui-Wang, Syed Y. Abbas, Neil L. Harrison, and Peter A. Goldstein. "Propofol block of $\mathrm{l}_{\mathrm{h}}$ contributes to the suppression of neuronal excitability and rhythmic burst firing in thalamocortical neurons." Eur. J. Neurosci. 23, no. 2 (2006): 465-480.

54. Ungless, Mark A., Jennifer L. Whistler, Robert C. Malenka, and Antonello Bonci. "Single cocaine exposure in vivo induces long-term potentiation in dopamine neurons." Nature 411, no. 6837 (2001): 583-587.

55. Mameli, Manuel, Bénédicte Balland, Rafael Luján, and Christian Lüscher. "Rapid synthesis and synaptic insertion of GluR2 for mGluR-LTD in the ventral tegmental area." Science 317, no. 5837 (2007): 530-533.

56. Wei, Chun-Ling, Zhi-Qiang Liu, Yi-Hui Liu, and Wei Ren. "High-frequency stimulation on cell soma induces potentiation of intrinsic excitability in VTA dopaminergic neurons." Sheng li xue bao:[Acta physiologica Sinica] 65, no. 1 (2013): 55-60.

57. Grace, Anthony A., and Benjamin S. Bunney. "The control of firing pattern in nigral dopamine neurons: burst firing." Journal of neuroscience 4, no. 11 (1984): 2877-2890.

58. Tsai, Hsing-Chen, Feng Zhang, Antoine Adamantidis, Garret D. Stuber, Antonello Bonci, Luis De Lecea, and Karl Deisseroth. "Phasic firing in dopaminergic neurons is sufficient for behavioral conditioning." Science 324, no. 5930 (2009): 1080-1084.

59. Mikhailova, Maria A., Caroline E. Bass, Valentina P. Grinevich, Ann M. Chappell, Alex L. Deal, Keith D. Bonin, Jeff L. Weiner, Raul R. Gainetdinov, and Evgeny A. Budygin. "Optogenetically-induced tonic dopamine release from VTA-nucleus accumbens projections inhibits reward consummatory behaviors." Neuroscience 333 (2016): 54-64.

60. Cooper, Donald C. "The significance of action potential bursting in the brain reward circuit." Neurochemistry international 41, no. 5 (2002): 333-340.

61. Zeldenrust, Fleur, Wytse J. Wadman, and Bernhard Englitz. "Neural coding with bursts—current state and future perspectives." Frontiers in computational neuroscience 12 (2018): 48.

62. Oster, Andrew, Boris Gutkin, and Philippe Faure. "Mechanisms for multiple activity modes of VTA dopamine neurons." Front. Comp. Neurosci. 9 (2015): 95.

63. Borgland, Stephanie L., Robert C. Malenka, and Antonello Bonci. "Acute and chronic cocaine-induced potentiation of synaptic strength in the ventral tegmental area: electrophysiological and behavioral correlates in individual rats." J. Neurosci. 24, no. 34 (2004): 7482-7490.

64. Bocklisch, Christina, Vincent Pascoli, Jovi CY Wong, David RC House, Cédric Yvon, Mathias De Roo, Kelly R. Tan, and Christian Lüscher. "Cocaine disinhibits dopamine neurons by potentiation of GABA transmission in the ventral tegmental area." Science 341, no. 6153 (2013): 1521-1525.

65. Wu, Xing, Liping Liao, Xiangming Liu, Fang Luo, Tianming Yang, and Chenhong Li. "Is ZD 7288 a selective blocker of hyperpolarization-activated cyclic nucleotide-gated channel currents?." Channels 6, no. 6 (2012): 438-442.

66. Chen, Xiangdong, Shaofang Shu, Dylan P. Kennedy, Sarah C. Willcox, and Douglas A. Bayliss. "Subunitspecific effects of isoflurane on neuronal I h in HCN1 knockout mice." Journal of neurophysiology 101, no. 1 (2009): 129-140.

67. Marinelli, M., C. N. Rudick, X-T. Hu, and F. J. White. "Excitability of dopamine neurons: modulation and physiological consequences." CNS \& Neurological Disorders-Drug Targets (Formerly Current Drug Targets-CNS \& Neurological Disorders) 5, no. 1 (2006): 79-97.

68. Pan, Wei-Xing, Robert Schmidt, Jeffery R. Wickens, and Brian I. Hyland. "Dopamine cells respond to predicted events during classical conditioning: evidence for eligibility traces in the reward-learning network." Journal of Neuroscience 25, no. 26 (2005): 6235-6242.

69. Hyland, Brian I., J. N. J. Reynolds, J. Hay, C. G. Perk, and R. Miller. "Firing modes of midbrain dopamine cells in the freely moving rat." Neuroscience 114, no. 2 (2002): 475-492.

70. Arencibia-Albite, Francisco, Carlos Paladini, John T. Williams, and Carlos A. Jiménez-Rivera. "Noradrenergic modulation of the hyperpolarization-activated cation current $\left(\mathrm{I}_{\mathrm{h}}\right)$ in dopamine neurons of the ventral tegmental area." Neuroscience 149, no. 2 (2007): 303-314.

71. Paxinos, G., and Ch Watson. "The rat brain in stereotaxic coordinates: compact sixth edition." (2009). 
689

690

691

692

693

694

695

696

697
72. Margolis, Elyssa B., Hagar Lock, Gregory O. Hjelmstad, and Howard L. Fields. "The ventral tegmental area revisited: is there an electrophysiological marker for dopaminergic neurons?." The Journal of physiology 577 , no. 3 (2006): 907-924.

73. Margolis, Elyssa B., Hagar Lock, Vladimir I. Chefer, Toni S. Shippenberg, Gregory O. Hjelmstad, and Howard L. Fields. "K opioids selectively control dopaminergic neurons projecting to the prefrontal cortex." Proceedings of the National Academy of Sciences 103, no. 8 (2006): 2938-2942.

74. Hopf, F. Woodward, Miquel Martin, Billy T. Chen, M. Scott Bowers, Maysha M. Mohamedi, and Antonello Bonci. "Withdrawal from intermittent ethanol exposure increases probability of burst firing in VTA neurons in vitro." Journal of neurophysiology 98, no. 4 (2007): 2297-2310. 